\title{
Simulation and Experimental Investigation on Micro Electrochemical Drilling of Deep Micro-Holes by using High-speed Spiral Electrode
}

\author{
Yong Liu, Chunsheng Guo \\ Associated Engineering Research Center of Mechanics and Mechatronic Equipment, Shandong \\ University, Weihai 264209, P.R.China \\ rzliuyong@163.com,guo@sdu.edu.cn
}

Keywords: High speed, Micro spiral electrode, Electrochemical drilling, Gas film, Deep micro-holes.

Abstract: In order to realize the fabrication of deep micro-holes, an electrochemical drilling process with high-speed micro spiral electrode is introduced. Firstly, the numerical simulation of the gap flow field was carried out. The simulation results show that with the increase of the electrode rotating speed, the gas film with good insulation effect around the electrode increases rapidly, which can eliminate the taper and improve the machining localization greatly. At the same time, with the increase of the electrode rotating speed, the enhanced mass transfer is performed well, which can improve the machining efficiency significantly. Secondly, the influences of predominant parameters such as rotating speed, feed rate, applied voltage and pulse frequency on machining localization were analyzed. Finally, the deep micro-holes with no taper, which the diameter is less than $400 \mu \mathrm{m}$, the aspect ratio is more than 10 , were fabricated successfully on the nickel-based super alloy.It is proved that the micro electrochemical drilling process with high-speed spiral electrode for fabricating deep micro-holes has a huge potential and broad application prospects.

\section{Introduction}

Deep micro-holes is an important structure which is widely used in fields of engineering related to aerospace and medical science, such as deep micro-holes in nickel-based alloy blade of aircraft engine. Currently micro-hole machining methods on the difficult-to-cut metals include: electro discharge machining, laser beam machining, electron beam machining and electrochemical micromachining (Mcgeough, 2001). However, because EDM, LBM and EBM are thermal machining process, it may cause deterioration of the machined surface, such as the generation of a heat-affected layer and many micro cracks. In the meantime, by reason of high cost, EBM is confined for further application.

EMM is a non-thermal machining process with no heat-affected layer. Moreover, it has many other advantages, such as, no tool wear, stress free, smooth surfaces, high efficiency, etc. In the past few years, scholars all over the world have made a lot of achievements in EMM(Rajurkar, 2006). M. Kock in Germany successfully machined a spiral trough with a depth of 5 um using the ultra-short 
voltage pluses in electrochemical machining(Kock, 2003). Tsuneo Kurita in Japan successfully machined a three-dimensional shape with sub-millimeter range using an electrochemical micromachining system developed with a machining gap control system(Kurita, 2006). B. H. Kim in Korea successfully machined some micro holes $(\phi 15 \mu \mathrm{m}, 50 \mu \mathrm{m}$ depth) into 304SS(Kim, 2005). A micro hole with diameter of about $70 \mu \mathrm{m}$ were machined by a $\phi 60 \mu \mathrm{m}$ spherical electrode on a more than $200 \mu \mathrm{m}$ thick stainless steel plate by Liu(Liu, 2015).

However, normal EMM method is unable to meet the requirements of machining deep microholes. As the micro hole becomes deeper, the removal of the machining by-products and the replenishment of the electrolyte become difficult. It may cause short-circuit and the suspension of the machining. In this paper, an electrochemical drilling method for fabricating deep micro-holes was proposed. High-speed rotating micro spiral electrode and microsecond pulse power are combined to help remove by-products and replenish electrolyte, and to meet the requirement of the deep micro-holes machining. First, the numerical simulation of the gap flow field was simulated by FLUENT. By changing the rotating speed of the spiral electrode, the water/gas distribution and the flow velocity vector diagrams in the machining gap under different rotary speeds could be obtained. Second, the simulation results above were verified by the experiments, The influences of some predominant parameters such as rotating speed, applied voltage, pulse frequency, and feed rate on machining localization were analyzed. Finally, a row of deep micro-holes, which the diameter is less than $400 \mu \mathrm{m}$, the aspect ratio is more than 10 , and has steep wall and the sharp edge were fabricated successfully on the nickelbase superalloys, which is proved the high-speed electrochemical drilling process for fabricating deep micro-holes has a huge potential and broad application prospects.

\section{Principle and Simulation}

\subsection{Principle of Micro Electrochemical Drilling}

Electrochemical drilling is an electrochemical anodic dissolution process in which a direct ultrashort pulse current with low voltage is passed between workpiece and cathode. The conductive material is used as anode and the micro electrode is used as cathode. The anode workpiece will be dissolved into metallic ions under the reaction of electrochemistry and to be fabricated as target structure. The schematic diagram of electrochemical drilling is shown as Figure 1.

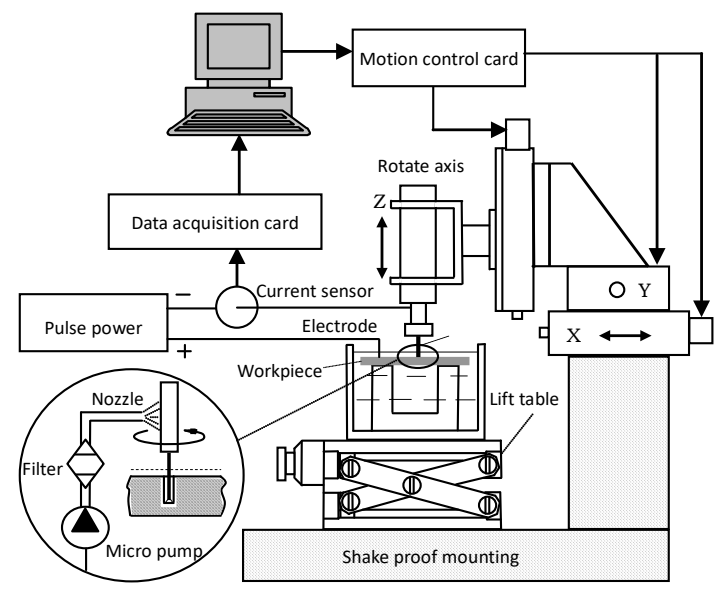

Figure 1 This caption has one line so it is centered.

During the machining process, the high-speed electricity spindle drives the cathode to rotate at a high speed, with the high frequency pulse direct current passing between workpiece and cathode. 
With the increase of the electrode rotating speed, the gas film with good insulation effect around the electrode increases rapidly, which can reduce or even eliminate the taper of the hole, then improve the machining localization greatly.

When the machining gap (includes the end gap and the side gap) becomes as small as $5 \sim 10 \mu \mathrm{m}$, the removal of the machining by-products and the replenishment of the electrolyte in the normal EMM system become difficult, or almost impossible. To resolve this issue, we use high-speed rotating (the maximum speed is as large as $50000 \mathrm{rpm}$ ) micro spiral electrode as the cathode. During the machining process, the machining gap is filled with electrolyte, and the cathode rotates at a high speed which can help improve the removal of the by-products and the replenishment of the electrolyte.

\subsection{Simulation of Field in Machining Gap}

In order to study the influences of high speed micro spiral electrode on machining localization and machining efficiency, the numerical simulation of the gap flow field are carried out. the numerical simulation of the gap flow field was simulated by FLUENT. By changing the rotating speed of the spiral electrode, the water/gas distribution and the flow velocity vector diagrams in the machining gap under different rotary speeds could be obtained.

\subsubsection{Computational Model and Boundary Conditions}

Assuming that the electrolyte is a constant incompressible Newton fluid, the air satisfies the ideal gas state equation. Considering a problem of turbulent isothermal gas-liquid two-phase flow with the continuous liquid phase and the dispersed gas phase in the three-dimensional (3D) electrolytic cell, the air and the electrolyte are respectively followed by mass conservation equation and momentum conservation equation, and the two phases is the volume of fluid (VOF) model. The VOF model is a surface-tracking technique applied to a fixed Eulerian mesh. In the present study, the computation fluid dynamic (CFD) solver FLUENT solves the incompressible flow of the 3D Reynolds averaged Navier Stokes equation. Use the control volume method to solve the governing equations. The governing equations are integrated in each control volume to construct the dependent variables of the discrete algebraic equations. The convective flux of averaged volume fraction, momentum and turbulence closure equations are discretized using a conservative, secondorder accurate quick scheme. The pressure velocity coupling algorithm is the pressure-based implicit splitting of operators (PISO) method, which is based on the approximate relationship between the pressure and velocity corrections to a higher degree.

Mohamed et al. pointed out that the smaller domains are associated with a large variation of the rotor coefficient19. This suggests that the computational domain should be extended to the rotor radius of at least 20 times in each direction. Therefore, the computational domain as shown Figure 2 has been retained for all further computations in this paper.

In this paper, the free surface of the electrolyte tank was specified as slip wall, which is zero shear stress boundary. The initial height of free surface is located at $0.3 \mathrm{~mm}$ above the surface of the workpiece. Other boundary settings are as follows:

Boundary1,2,3,4= no-slip smooth walls

Boundary5= no-slip rotating wall

The electrode rotation speeds are set to 10000, 20000, 30000, 40000, 45000 and 50000rpm.

Fig.2-a) shows the 3D computational domain of two cylinders with communications. The small cylinder shows the removed part of the workpiece, and has a diameter of $2 \mathrm{~mm}$ and a height of $1.5 \mathrm{~mm}$. The large cylinder indicates a portion of the electrolyte, and has a diameter of $0.3 \mathrm{~mm}$ and a height of $0.5 \mathrm{~mm}$. As Fig.2-b) shows, the electrode is located in the center of the computational 
domain; the diameter of the electrode is $0.2 \mathrm{~mm}$. The space below the gap and the lateral clearance between the end of the electrode and the face of specimens measure $0.1 \mathrm{~mm}$ and $0.05 \mathrm{~mm}$ respectively.

As shown in Fig.2-c), the mesh had unstructured grids, which contains tetrahedral, pyramid and prismatic elements. Various aspects of the mesh that affect the flow field close to the electrode, are evaluated.

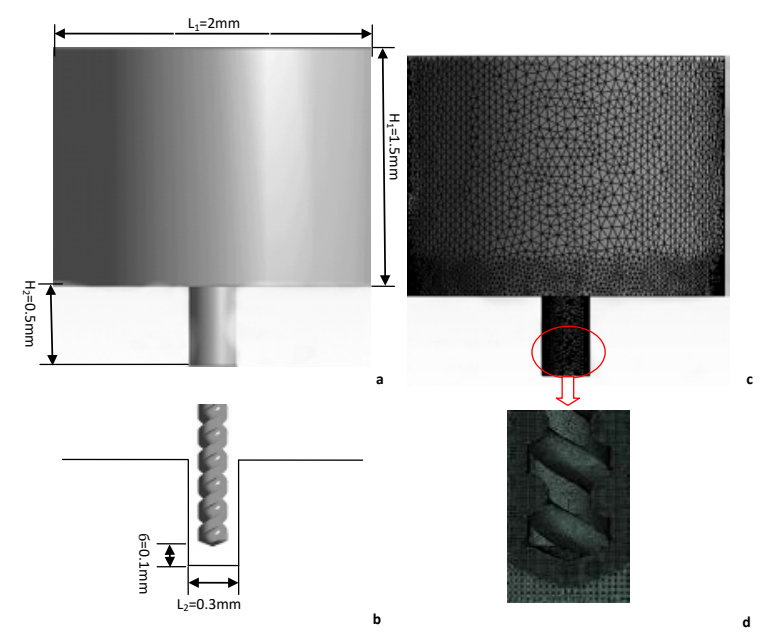

Figure 2 CFD model geometry and mesh.

\subsubsection{Effects of Electrode Rotation Speed on Gas Distribution}

The electrolyte volume fraction is characterized by the number of electrolyte per unit volume. Figure 3 shows the axial sections of model, which depicts the electrolyte volume fraction for different rotation speeds, 10000 rpm, 20000rpm, 30000 rpm, 40000 rpm, 45000rpm and 50000rpm. Obviously, the increase of electrode rotation speed leads to decrease in electrolyte volume fraction of the machining gap. The predicted cloud charts of electrolyte volume fraction clearly also show inward movement of gas while rising rotation speed, and then gas-electrolyte two-phase mixing layer, which is cling to electrode, is formed gradually. This trend is slowness for rotation speed over $40000 \mathrm{rpm}$. The gas-electrolyte two-phase mixing layer is beneficial to reduce the current density along the sidewall of gap. Moreover, from Fig.3 can also be found that thinness of gas-electrolyte two-phase mixing layer along the sidewall of gap is similar. This shows that the current density is evenly along the sidewall of gap.

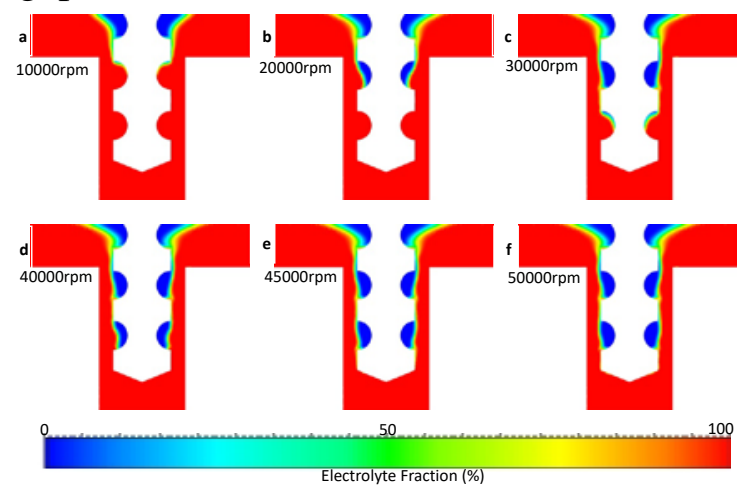

Figure 3 Electrolyte volume fraction contours on the axial sections of model. 


\section{Results and Discussions}

In order to verify the simulation results above, the influences of some predominant parameters such as rotating speed, feed rate, applied voltage and pulse frequency on machining localization were analyzed by sets of experiments.

\subsection{Effect of Rotating Rate on Side Gap}

When the other parameters keep unchanged, the basic machining conditions are as follows: machining voltage of $12 \mathrm{v}, 14 \mathrm{v}, 16 \mathrm{v}$ respectively, pulse width of $100 \mu \mathrm{s}$, pulse frequency of $1 \mathrm{KHZ}$, tool electrode of 200 microns in diameter, the relationships between the side gap and the rotation speed as shown in Figure 4. Three squares curve shows that with the electrode speed from 0 to $10000 \mathrm{rpm}$, the side gap has the obvious upward trend; Varying from 10000 to $20000 \mathrm{rpm}$, the side gap slowly rises; After more than $20000 \mathrm{rpm}$, the side gap has slightly downward trend. When rotation speed of electrode achieve $40000 \mathrm{rpm}$, the side gap remains stable and keeps almost the same.

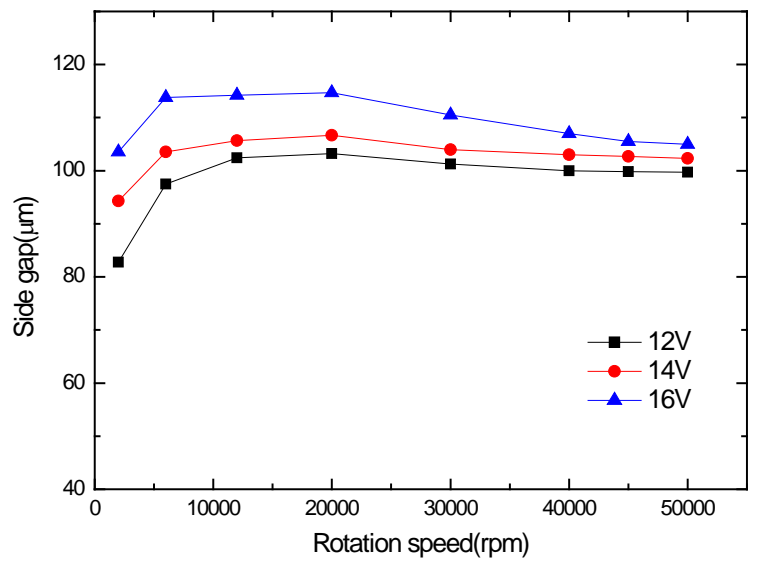

Figure 4 Relationships between side gap and electrode speed.

This experiments show that, with the increase of the rotate speed, the speed of vertical vortex gets increased. The vacuum suction makes the by-product remove along the vertical vortex out of the machining gap. In the meantime, the fresh electrolyte was engulfed into the machining gap. With the sustained increase of the rotate speed (more than 20000rpm), the insulation of the gas film began to become more and more obvious, the side gap decreases slowly, and the machining stability increases gradually.

Known from the analysis of the above, the conditions of high speed, smaller voltage, the appropriate pulse frequency are conducive to enhancing the machining localization of electrochemical drilling process.

\subsection{Effect of Feed Rate on Side Gap}

Figure 5 shows the relationships between feed rate and side gap, under the machining condition of pulse width of $100 \mu \mathrm{s}$, pulse frequency $1 \mathrm{KHZ}$, rotate speed of 40000rpm, the machining voltage of $14 \mathrm{~V}$ and the feed rate varies from 1 to $15 \mu \mathrm{m}$.

With the optimum rotate speed of the electrode, as the feed speed of the cathode increases within a certain range, the acting time of the secondary electrolysis decreases, so the side gap becomes smaller, and the machining localization and the machining efficiency get better. 


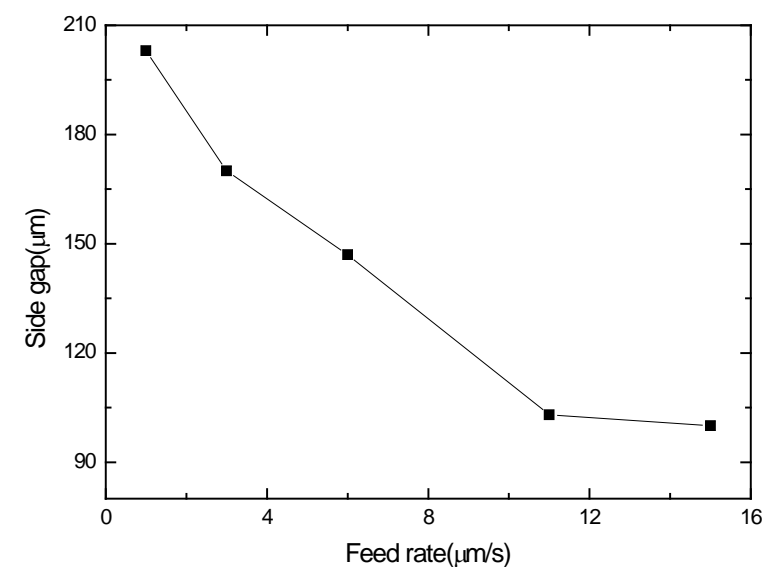

Figure 5 Relationships between feed rate and side gap.

\subsection{Effect of Applied Voltage on Side Gap}

The basic machining conditions of this set of experiments were almost the same as above: pulse width of $100 \mu \mathrm{m}$, impulse frequency of $1 \mathrm{kHz}$, rotate speed of the electrode 40000rpm and applied voltages varies from 10 to 20V. Figure 8 shows the relationships between the side gap and applied voltage.

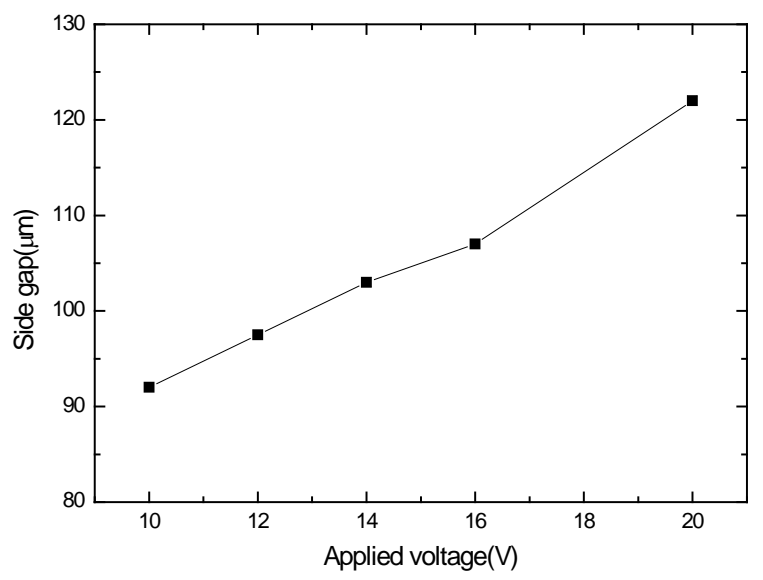

Figure 6 Relationships between applied voltage and side gap.

As can be seen from the relation curves, the side gap increase with the increasing voltage. Experimental results reveal that if the feed rate remains unchangeable, the removal rate of the workpiece material increases with the machining voltage increases, and lead to the decrease of the machining localization. So when the voltage rises, the feed rate should be increased to meet the dynamic balance of the material removal rate and feed rate, and to form a new balance gap.

\subsection{Effect of Pulse Frequency on Side Gap}

Figure 7 shows the relationships between pulse frequency and side gap when the pulse width was $100 \mu \mathrm{s}$. The machining conditions were as follows: machining voltage $14 \mathrm{~V}$, rotate speed of 40000rpm and pulse frequency varies from 1 to 5kHZ. From the Fig. 7, we can see that side gap increases with the pulse frequency increasing. As the machining current between the cathode and anode is one of the key factors which influences the machining precision. As the pulse frequency rises, machining current gets increased. According to the Faraday's law, , when the removal of the workpiece material increases, the side gap increases and the machining localization decreases. 
Therefore, the smaller pulse frequency should be suggested to improve the machining localization.

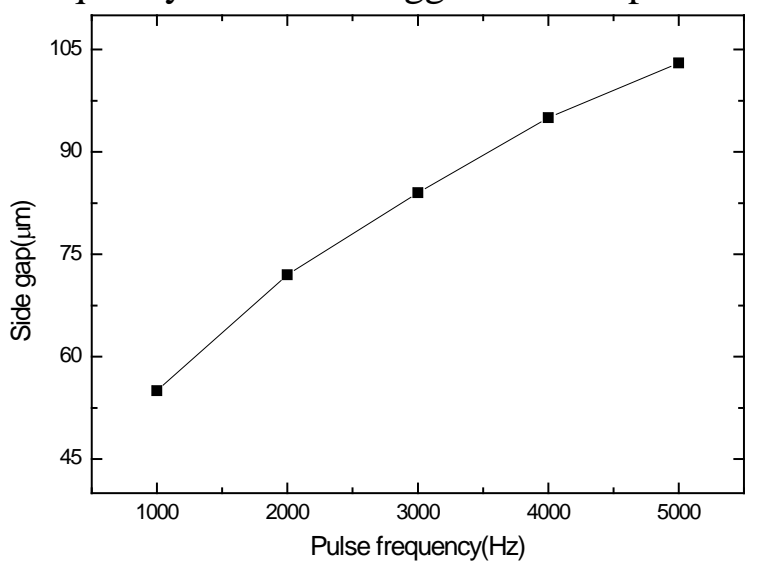

Figure 7 Relationships between pulse frequency and side gap.

\section{Experimental Results}

Figure 8 show five graphs of deep micro-holes of about $400 \mu \mathrm{m}$ in diameter, which fabricated under the same conditions of spiral electrode of 200 microns in diameter, voltage of $14 \mathrm{~V}$, electrode speed of $40000 \mathrm{rpm}$, pulse width of $100 \mu \mathrm{s}$, pulse frequency of $5 \mathrm{KHZ}$, electrolyte of $16 \% \mathrm{NaNO} 3$ solution, the feed rate of $11 \mu \mathrm{m} / \mathrm{s}$, and the initial gap of $5 \mu \mathrm{m}$ on a $4 \mathrm{~mm}$ thickness GH4169 plate. As shown in Fig.10(c), the diameters of above 5 deep micro-holes have good consistency. The diameters of the No.1-5 hole are $405 \mu \mathrm{m}, 398 \mu \mathrm{m}, 390 \mu \mathrm{m}, 395 \mu \mathrm{m}$ and $400 \mu \mathrm{m}$ repectively. All the deep micro-holes have almost no taper, and the aspect ratio is more than 10:1.

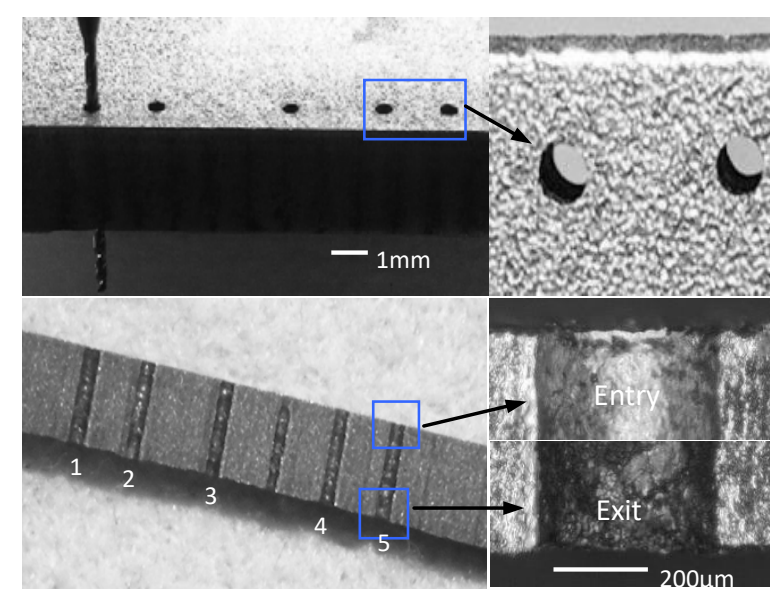

Figure 8 Deep micro-holes object.

\section{Conclusions}

The micro electrochemical drilling process with high speed spiral electrode was numerical analyzed and experimental study based on the mechanism analysis of the influences of main parameters on machining localization. The conclusions can be summarized as follows:

(1) With the increase of the electrode rotating speed, the gas film with good insulation effect around the electrode increases rapidly, which can eliminate taper of the micro hole and improve the machining localization greatly.

(2) Enhanced mass transfer is performed well with the increase of the electrode rotating speed, which can improve the machining efficiency significantly. The by-product in the machining gap 
was risen spirally along with the electrolyte and then thrown out quickly with the increase of the electrode rotary speed. At the same time, the fresh electrolyte outside is forced into the machining area due to the negative pressure formed under the electrode bottom.

(3) In order to obtain a good machining localization and efficiency, many suggested values of the key machining parameters are proposed, such as, high rotation speed, smaller voltage, the appropriate pulse frequency, and the higher feed rate.

(4) A set of deep micro-holes with the diameter of less than $400 \mu \mathrm{m}$, the aspect ratio of about 10 and no taper are fabricated successfully on the nickel-based super alloy, which is proved that the high-speed electrochemical drilling process for fabricating deep micro-holes has a huge potential and broad application prospects.

\section{Acknowledgements}

Authors acknowledge financial support from the National Natural Science Foundation of China (No. 51305238), the China Postdoctoral Science Foundation (No. 2015M572023), and the Shandong Provincial Natural Science Foundation (No. ZR2017BEE012), the Program of Science and Technology of Suzhou (No. 2017GHY15122) and the Young Scholars Program of Shandong University, Weihai (2015WHWLJH03).

\section{References}

[1] Mcgeough, J. A., Leu, M. C., Rajurkar, K. P., Silva, A. K. M. D., \& Liu, Q, 2001. Electroforming process and application to micro/macro manufacturing, CIRP Annals - Manufacturing Technology. 50(2), pp.499-514.

[2] Rajurkar, K.P., Levy, G., Malshe, A., Sundaram, M.M., Mcgeough, J.A., 2006. Micro and nano machining by electro-physical and chemical processes. CIRP Annals - Manufacturing Technology. 55 (2), pp.643-666.

[3] Kock, M., Kirchner, V., \& Schuster, R.,2003. Electrochemical micromachining with ultrashort voltage pulses-a versatile method with lithographical precision, Electrochimica Acta. 48(20), pp.3213-3219.

[4] Kurita, T., Chikamori, K., Kubota, S., \& Hattori, M., 2006. A study of three-dimensional shape machining with an ecum system, International Journal of Machine Tools \& Manufacture. 46(12-13), pp.1311-1318.

[5] Kim, B. H., Na, C. W., Lee, Y. S., Choi, D. K., \& Chu, C. N., 2005. Micro electrochemical machining of 3d micro structure using dilute sulfuric acid, CIRP Annals - Manufacturing Technology. 54(1), pp.191-194.

[6] Liu, Y., Cai, H., \& Li, H., 2015. Fabrication of micro spherical electrode by one pulse edm and their application in electrochemical micromachining, Journal of Manufacturing Processes. 17, pp.162-170. 